\title{
Dependence of star formation on initial conditions and molecular cloud structure
}

\author{
Matthew R. Bate \\ School of Physics, University of Exeter, \\ Stocker Road, Exeter EX4 4QL, United Kingdom \\ email: mbate@astro.ex.ac.uk
}

\begin{abstract}
I review what has been learnt so far regarding the origin of stellar properties from numerical simulations of the formation of groups and clusters of stars. In agreement with observations, stellar properties are found to be relatively robust to variations of initial conditions in terms of molecular cloud structure and kinetics, as long as extreme initial conditions (e.g. strong central condensation, weak or no turbulence) and small-scale driving are avoided, but properties may differ between bound and unbound clouds. Radiative feedback appears crucial for setting stellar masses, even for low-mass stars, while magnetic fields can provide low star formation rates.
\end{abstract}

Keywords. gravitation; hydrodynamics; magnetic fields, MHD; radiative transfer; stellar dynamics; methods: numerical; stars: formation; stars: low-mass, brown dwarfs; stars: luminosity function, mass function

\section{Introduction}

The star formation process is complex, involving a huge range of spatial scales (roughly 10 orders of magnitude from $\sim 10$ pc to $\sim \mathrm{R}_{\odot}$ ) and temporal scales (roughly 12 orders of magnitude, from several million years to a few minutes, the latter being the time required for a sound wave to propagate across the Sun). It also involves a large number of physical processes, including gravity, supersonic fluid dynamics, radiative transfer, magnetic fields, and chemistry. No numerical simulation can include all of this complexity currently.

To simulate the formation of a star, the bare minimum of physics required even to get started is self-gravitating compressible fluid dynamics. The first calculations of star formation were performed more than 40 years ago by Larson (1969). These one-dimensional calculations also included radiative transfer. Soon after, hydrodynamical calculations were extended to two dimensions (e.g. Larson 1972) and three dimensions (e.g. Boss \& Bodenheimer 1979). However, until the late 1990s, most calculations were limited to investigating the formation of single stars or small multiple systems (e.g. binary or triple systems) within isolated molecular cloud cores (e.g. Boss 1986; Bonnell et al. 1991).

However, the three-dimensional calculations of even these relatively simple systems could not be followed very far in time because as the collapse to form the first star occurred, the timesteps required to evolve the calculation decreased by orders of magnitude. Thus, each calculation would grind to a halt as the first star forms. Order to get past this point, an approximation must be made. The standard procedure now used in both particle-based (Bate, Bonnell \& Price 1995) and grid-based numerical methods (Krumholz, McKee \& Klein 2004) is to replace collapsing protostars by sink particles. Here the dense gas within a specified radius of the centre of the protostar, the accretion radius, is combined into a single point mass with the same total mass and momentum as the gas it replaces. Gas that subsequently falls within this radius is accreted by the sink particle. This method allows the very short timesteps that would be required to 
evolve the gas deeper inside the protostar to be avoided, so hydrodynamical calculations can be evolved well beyond the collapse to form the first protostar. The sink particle method opened the way for large-scale hydrodynamical calculations of stellar groups and clusters to be performed, since the evolution of a molecular cloud could be followed over its dynamical time (typically hundreds of thousands to millions of years) at the same time as resolving small scales (down to the sink particle accretion radii).

The first hydrodynamical calculations of the formation of groups or clusters of stars began with those of Chapman et al. (1992), who studied star formation due to colliding molecular clouds (without sink particles), and Klessen, Burkert \& Bate (1998) who studied the collapse and fragmentation of a clumpy molecular cloud to form 55 protostars (modelled by sink particles) and obtained an approximately log-normal mass function. At the same time, Bonnell et al. (1997) used sink particles to model the growth of protostellar 'seeds' as they accreted from a molecular cloud and found that the stellar mass distribution resulted from 'competitive accretion' between the protostars. Subsequent calculations showed that the peak of the mass function obtained from such calculations was located near the mean Jeans mass of the initial clouds (Klessen \& Burkert 2000, 2001; Bate, Bonnell \& Bromm 2003; Bate \& Bonnell 2005).

The ability, over the past decade, to begin simulating the formation of clusters of stars directly using self-gravitating hydrodynamical calculations has opened up the possibility of trying to understand the origin of the statistical properties of stellar systems by conducting 'numerical experiments'. In these experiments, the initial conditions for star formation and/or physical processes included are varied and the effect of these changes on the outputs of the star formation process (i.e. the statistical properties of the stellar systems) are measured. In this way, we can hope to learn what conditions and processes are important for determining stellar properties, and which have only second-order effects.

\section{Hydrodynamical simulations}

Despite the use of sink particles, many hydrodynamical calculations of star formation published to date only resolve the collapsing protostars to scales of several hundreds of AU (i.e. the sink particles had accretion radii of hundreds of AU). Thus, they do not capture the opacity limit for fragmentation that occurs when collapsing gas becomes optically thick to its own radiation and so do not capture all of the expected fragmentation. This leads to incomplete mass functions, with the lowest mass objects (e.g. brown dwarfs) missing and, presumably, affects the higher mass protostars as well because there are fewer objects accreting competitively. Most stellar multiple systems and discs are also unresolved. This severely limits the comparisons that can be made with observations.

The first cluster formation calculation that resolved the opacity limit for fragmentation, thus capturing all fragmentation and resolving even the lowest mass brown dwarfs, was that of Bate, Bonnell \& Bromm (2002a,b, 2003). The calculation began with a uniform sphere of molecular gas at a temperature of $10 \mathrm{~K}$. Structure was generated in the gas by imposing an initial solenoidal random Gaussian velocity field on the gas to mimic the turbulence that is observed to be present in molecular clouds. These 'turbulent' motions were imposed initially and then allowed to decay during the calculation; the turbulence was not 'driven'. The opacity limit for fragmentation was modelled using a barotropic equation of state in which the gas temperature increased with the gas density once the gas once the gas began to trap its own radiation. The calculation also resolved binaries with separations as close as $1 \mathrm{AU}$ and discs with radii down to $\approx 10 \mathrm{AU}$. The calculation demonstrated that star formation in clusters could be highly chaotic and dynamic, with discs being truncated by dynamical encounters, fragmention to form multiple systems, 
and stars and brown dwarfs ejected from unstable multiple systems and escaping the cluster. The calculation produced a modest 50 stars and brown dwarfs, enough for a crude comparison with observations. Properties such as stellar multiplicity, and the fraction of close binaries, were in agreement with observations while the initial mass function (IMF) was in qualitative agreement, though over produced brown dwarfs. As with the earlier, more poorly resolved, simulations, the stellar masses were found to originate through a process of competitive accretion with the characteristic stellar mass being roughly the Jeans mass. All objects began with low masses and accreted from the cloud, typically to the mean thermal Jeans mass, but with a few stars in the centres of sub-clusters, reaching higher masses and forming the high-mass end of the IMF. For those objects with final masses much less than the typical Jeans mass (i.e. brown dwarfs), dynamical interactions and ejections from multiple systems were the key to their low masses (Bate et al. 2002a) as proposed by Reipurth \& Clarke (2001). Brown dwarfs began with low masses (as did those objects that ended up with stellar masses), but their accretion was terminated when they were involved in dynamical interactions in small groups which increased their velocities (but typically only to a few $\mathrm{km} / \mathrm{s}$ ) and ejected them from the dense cores in which they began forming. Thus, they were unable to accrete to the typical Jeans mass and ended up with substellar masses. Approximately 3/4 of the brown dwarfs were found to originate from the fragmentation of massive circumstellar discs, while the remainder formed in dense filaments, fell into existing multiple systems and then were ejected.

\subsection{The dependence of star formation on the Jeans mass}

This first hydrodynamical calculation of star cluster formation that resolved the opacity limit for fragmentation was followed by three similar calculations that investigated the dependence of stellar properties on the initial conditions in the molecular clouds and variations in the opacity limit for fragmentation. Bate \& Bonnell (2005) performed an identical calculation to that of Bate et al. (2003), but for a cloud with a smaller radius and nine times higher density. Thus, the mean thermal Jeans mass in the cloud was $1 / 3$ of that in the original calculation. The calculation produced a median stellar mass a factor of 3 lower than the original calculation, exactly matching the change in the mean Jeans mass. This confirmed the results of the earlier, more poorly resolved, calculations mentioned above which indicated that the typical stellar mass was similar to the mean thermal Jeans mass. More recently, Jappsen et al. (2005) and Bonnell, Clarke \& Bate (2006) using calculations with non-isothermal equations of state at very low molecular densities showed that the transition from atomic line to dust cooling could set an appropriate Jeans mass which in turn could produce the characteristic mass of the IMF.

\subsection{The dependence on the opacity limit for fragmentation}

Bate (2005) performed a third opacity limited calculation, identical to that of Bate et al. (2003) except that the opacity limit for fragmentation was moved to a lower density by a factor of nine (i.e. the transition from an isothermal collapse to the gas becoming optically thick to its own radiation was assumed to occur at earlier in the collapse). This increased the minimum mass by a factor of 3 to $\approx 9$ Jupiter masses. Such a change may occur, for example, in lower metallicity gas which cools less effectively. Bate found that apart from increasing the minimum mass of a brown dwarf, this change to the equation of state produced no large change in the rest of the IMF or the other stellar properties.

\subsection{The dependence on turbulent motions and molecular cloud structure}

Bate (2009c) performed a fourth opacity limited calculation, identical to Bate et al. (2003) except the power spectrum of the initial turbulent velocity field was $P(k) \propto$ 
$k^{-6}$ to give more power on large scales, rather than $P(k) \propto k^{-4}$ (which was chosen to match the observed Larson (1981) scaling relations). The structures produced in the cloud during the evolution were very different to those found in the original calculation with large shocks and little small-scale structure. However, despite this difference, the stellar properties obtained were indistinguishable from those of the original calculation. In particular, the IMFs were almost identical.

Other studies have also investigated the dependence of the star formation on the properties of the turbulence, though these calculations have not resolved the opacity limit for fragmentation. For example, another approach is to use a periodic box and drive turbulence (in Fourier space), only turning on gravity once the turbulence attains a quasi-steady state. Neither this nor the above approach is physically consistent and it is unclear which is more realistic. Klessen (2001) investigated clouds with driven turbulence and found that the resulting stellar mass function was broader and flatter if the turbulence was driven on small scales than for large-scale driving. More recently, Offner et al. (2008) showed that the outcomes of the star formation process does not depend greatly on whether decaying turbulence or large-scale driving are used.

There is also the question of the magnitude of the turbulence. The above calculations assumed that the energy of the turbulent motions is approximately equal to the gravitational potential energy of the clouds. Of course, in the extreme case of no motions, such uniform spherical initial conditions would collapse to a single massive protostar. Similarly, if the initial conditions are strongly centrally condensed, this favours the formation of a single massive object rather than fragmentation into many objects (e.g. Dobbs, Bonnell, Clark 2005; Krumholz, Klein, McKee 2007; Girichidis et al. 2010). On the other hand, there is the question of how strongly centrally-condensed molecular clouds could arise in nature without collapse and fragmentation occurring while the object was being assembled. Furthermore, in order for an unusual IMF to be obtained, the object would need to be formed and collapse in relative isolation so that other low-mass stars did not form nearby and result in a more normal mass function overall. Except in strongly-centrally condensed initial conditions, hydrodynamical calculations usually find that star formation proceeds through the formation of multiple groups or sub-clusters. If the global cloud is bound, these sub-clusters typically fall together and merge into larger clusters. Thus, large stellar clusters are thought to form hierarchically (Bonnell, Bate \& Vine 2003).

If the magnitude of the turbulence is such that the cloud is globally unbound, however, the star-forming groups and sub-clusters will not merge into a large bound cluster. Stars will still form from dense filaments and other structures formed by colliding flows, but the efficiency with which the gas is converted to stars is decreased as the level of turbulence increases (Clark \& Bonnell 2004; Clark et al. 2005). Recently, Bonnell et al. (2010) has also shown that the IMF may also vary between global bound and globally unbound star-forming regions with star formation in unbound regions resulting in fewer brown dwarfs and high-mass stars relative to solar-type stars than are found in bound regions.

\subsection{Comparison of hydrodynamical calculations with observed stellar properties}

The above calculations either produced only a few dozen objects, or did not resolve the opacity limit for fragmentation, brown dwarfs, or many multiple systems. Thus, detailed comparison with the observed properties of stars and brown dwarfs was not possible.

Recently, Bate (2009a) performed a hydrodynamical simulation of star cluster formation that resolved the opacity limit for fragmentation and produced well over 1000 stars and brown dwarfs. The calculation was identical to that of Bate et al. (2003), except that the cloud was an order of magnitude more massive $\left(500 \mathrm{M}_{\odot}\right)$. With so many objects, the statistical properties of low-mass stars and brown dwarfs are well determined 
and detailed comparison with observed stellar properties is possible. Bate found that many stellar properties were in good agreement with observations. For example, stellar multiplicity was found to be a strongly increasing function of primary mass with the multiplicity of very-low-mass objects $\left(0.03-0.10 \mathrm{M}_{\odot}\right)$, M-dwarfs, and solar-type stars increasing from $\approx 20$ to $60 \%$ as observed. The trends for low-mass binaries to have smaller separations and equal-mass components were also reproduced, and even the distribution of relative orientations of the orbital planes of triple systems were found to be in agreement with the observed distribution. The two main areas of disagreement with observed stellar properties were that the calculation produced a much higher ratio of brown dwarfs to stars than is observed, and there was a deficit of unequal-mass solar-type binaries.

\subsection{Conclusions based on hydrodynamical simulations}

The fact that numerical calculations only including gravity and fluid dynamics (without the more complicated physics of radiative transfer, magnetic fields, and chemistry) can reproduce many of the observed statistical properties of stellar systems implies the origin of these stellar properties is primarily due to dissipative gravitational dynamics and may not be significantly altered by additional processes.

The hydrodynamical calculations also indicate that the resulting stellar properties do not vary greatly with variations in the initial conditions such as the metallicity (as long as dust is still the primary coolant at intermediate densities), the power spectrum of the initial velocity field, and or whether or not decaying turbulence or large-scale driving is used. This is because, so long as there is sufficient structure in the gas and the equation of state allows fragmentation to produce many objects that interact dynamically, the processes of competitive accretion (Bonnell et al. 1997) and dynamical interactions and ejections (Bate et al. 2003) do not depend significantly on the initial structure and turbulent motions in the molecular cloud. On the other hand, extreme initial conditions such as highly centrally-condensed molecular clouds, a very weak (or no) velocity field, or small-scale turbulent driving can lead to statistically different stellar mass distributions. However, it is difficult to see how such initial conditions would arise in nature.

The relative invariance of the IMF to many variations in initial conditions is consistent with the lack of variation in the observed IMF (e.g. Bastian, Covey \& Meyer 2010). However, the hydrodynamical calculations do leave us with one serious difficulty: the characteristic mass of the IMF obtained from hydrodynamical calculations is found to scale with the typical Jeans mass of the molecular cloud. One potential solution to this problem is that the typical Jeans mass in molecular clouds does not vary greatly with different environmental conditions as argued by Elmegreen, Klessen \& Wilson (2008). But there is also the question of what impact extending the calculations beyond simple self-gravitating hydrodynamics will have.

\section{Radiation hydrodynamical simulations}

Thus, the question moves on to what the role of additional physical processes is on the star formation process. Boss et al. (2000) first pointed out that a barotropic equation of state is a poor approximation to including radiative transfer. Bate $(2009 \mathrm{~b})$ recently repeated the two cluster formation calculations of Bate et al. (2003) and Bate \& Bonnell (2005), this time including a realistic equation of state and radiative transfer in the flux-limited diffusion approximation. The calculations modelled gas to within $0.5 \mathrm{AU}$ of each protostar and the energy released down to such scales, but did not include the radiative feedback coming from the stars themselves or discs within 0.5 AU. Despite this, the inclusion of radiative feedback from the forming protostars back into the cloud had a 
huge effect on the fragmentation. Massive circumstellar discs, in particular, were found to be much hotter and more resistant to fragmentation than in the barotropic calculations. The result was than in both calculations, the numbers of objects formed were reduced by a factor of $\approx 4-5$ from the original calculations. This in turn led to fewer dynamical interactions between objects and fewer ejections. Since objects ejected soon after they form end up as low-mass stars and brown dwarfs, the ratio of brown dwarfs to stars was reduced by the inclusion of radiative feedback, bringing the IMFs produced by the calculations into much better agreement with observations.

As mentioned in the previous section, using a barotropic equation of state the stellar IMF was found to scale with the mean Jeans mass of the clouds. However, with radiative transfer, the IMFs produced from the two clouds were found to be indistinguishable despite their differing mean Jeans masses. When each protostar forms, it heats the gas surrounding it, inhibiting fragmentation nearby (i.e. locally the 'effective' Jeans length and Jeans mass are increased). For a denser cloud, where the initial Jeans length and mass are smaller than for a less dense cloud, the local heating increases the 'effective' Jeans mass by a proportionally greater amount than in a lower-density cloud, largely erasing the differences in the 'effective' Jeans mass between the two clouds. Thus, Bate $(2009 \mathrm{~b})$ proposed that radiative feedback from protostars self-regulates star formation and erases, or severely weakens, the dependence of the IMF on the mean Jeans mass of the progenitor cloud. This may help to explain the observed invariance of the IMF.

Following these calculations, Offner et al. (2009) also performed radiation hydrodynamical calculations low-mass star formation. Their calculations were performed using adaptive mesh refinement (AMR) whereas Bate used smoothed particle hydrodynamics (SPH). Unlike Bate, Offner et al. included radiative feedback from the stellar objects themselves and their accretion luminosity, but they used much larger accretion radii of $\approx 130$ AU. Urban, Martel \& Evans (2010) also investigated the effects of protostellar heating on the star formation process, using a simplified method to calculate dust temperatures near protostars and, again, large sink particles $(\approx 150 \mathrm{AU})$. Despite the differences between the calculations, each of these studies found that radiative feedback dramatically reduced the number of objects formed relative to calculations performed using a barotropic equation of state and, therefore, that even in the case of low-mass star formation, it is crucial to include the effects of radiative feedback.

\section{Magnetohydrodynamical simulations}

Magnetic fields have long been recognised as a potentially important physical process in star formation. However, while their role in some aspects of star formation is clearly crucial (e.g. protostellar jets), their role in star cluster formation is less clear. Recent ideal magnetohydrodynamical (MHD) calculations have confirmed that magnetic fields can play a crucial role in protostellar disc formation (Price \& Bate 2007; Hennebelle \& Fromang 2008) and the fragmentation of isolated molecular cloud cores to form binary and multiple systems (Price \& Bate 2007; Hennebelle \& Teyssier 2008). However, their role in cluster formation has only begun to be investigated numerically.

Price \& Bate (2008) recently repeated the calculation of Bate et al. (2003), this time including magnetic fields of varying strengths (mass-to-flux ratios from 20 to 3) using the ideal MHD approximation. For magnetic fields where the ratio of gas to magnetic pressure was less than unity (i.e. plasma $\beta<1$ ) the results were substantially different to the hydrodynamic case. Anisotropic turbulent motions and column density striations aligned with the magnetic field lines were found, both of which have been observed in the Taurus molecular cloud (Goldsmith et al. 2008). Large-scale magnetically supported 
voids were also produced. The additional large-scale support provided by the magnetic field strongly suppressed collapse in the clouds, reducing the star formation efficiency and leading to a more quiescent mode of star formation. Price \& Bate found an indication that the relative formation efficiency of brown dwarfs was lower in the strongly magnetized runs due to a reduction in the importance of protostellar ejections.

Conversely, Li et al. (2010) recently argued from AMR calculations that included magentic fields and a prescription for outflows that magnetic fields and outflows lower the characteristic stellar mass which they defined as the location of the break in a powerlaw fit to the high-mass end of the IMF. However, as with Price \& Bate (2008), the statistics from these calculations are still relatively poor and the median stellar masses of Li et al.'s hydrodynamical and magnetohydrodynamical calculations are similar.

\section{Radiation magnetohydrodynamical simulations}

Most recently, Price \& Bate (2009) combined their treatments of magnetic fields and radation hydrodynamics to perform the first radiation magnetohydrodynamical calculations of star cluster formation. Again, the calculations were of small 50- $M_{\odot}$ molecular clouds as modelled by Bate et al. (2003), Bate (2009b), and Price \& Bate (2008).

The effects of radiative feedback and magnetic fields, found separately in the earlier studies, were combined in these calculations. The main effect of radiative feedback was to inhibit fragmentation on small scales, while the main effect of magnetic fields was to provide support to the low-density gas on large scales, decreasing the star formation rate. With strong magnetic fields and radiative feedback the net result was an inefficient star formation process with a star formation rate of $\approx 10 \%$ per free-fall time. This is much less than the rates found without magnetic fields (typically $\approx 50 \%$ per free-fall time) and approaches the observed rate of $\sim 3-6 \%$ (Evans et al. 2009). However, it is also important to note that the star formation efficiency also depends on other parameters such as the boundedness of the molecular cloud (Clark \& Bonnell 2004) and whether and how the turbulence is driven (Klessen 2001; Offner et al. 2009).

\section{Conclusions}

Calculations of star cluster formation that only take into account gravity and fluid dynamics can reproduce many observed properties of stellar systems, including various binary properties. Many properties do not appear to depend sensitively on the properties of the molecular cloud structure and turbulence as long as extreme initial conditions are avoided (e.g. no motions or strongly centrally-condensed clouds) and the turbulence is decaying or driven on large scales (as seems to be observed; Brunt, Heyer \& Mac-Low 2009). This is due to the nature of competitive accretion and dynamical interactions between protostars which determine the spectrum of stellar masses in such calculations. These are local processes that have little memory of the large-scale initial conditions.

An exception is that, with only self-gravitating hydrodynamics, the characteristic mass of the IMF is found to scale linearly with the typical Jeans mass in the molecular cloud. The IMF may also differ in unbound clouds. Radiative feedback, even from low-mass protostars, appears to be crucial to obtain quantitative agreement with the observed IMF and may help to weaken the dependence of the IMF on the typical Jeans mass. Dynamically important magnetic fields also seem to be required to explain the low rate of star formation and many of the structures observed in molecular clouds. 


\section{References}

Bastian, N., Covey, K. R., \& Meyer, M. R. 2010, arXiv, 1001.2965

Bate, M. R. 2005, MNRAS, 363, 363

Bate, M. R. 2009a, MNRAS, 392, 590

Bate, M. R. 2009b, MNRAS, 392, 1363

Bate, M. R. 2009c, MNRAS, 397, 232

Bate, M. R. \& Bonnell, I. A., 2005, MNRAS, 356, 1201

Bate, M. R., Bonnell, I. A., \& Bromm, V. 2002a, MNRAS, 332, L65

Bate, M. R., Bonnell, I. A., \& Bromm, V. 2002b, MNRAS, 336, 705

Bate, M. R., Bonnell, I. A., \& Bromm, V. 2003, MNRAS, 339, 577

Bonnell, I. A., Bate, M. R., Clarke, C. J., Pringle, J. E. 1997, MNRAS, 285, 201

Bonnell, I. A., Bate, M. R., \& Vine, S. G. 2003, MNRAS, 343, 413

Bonnell, I. A., Clarke, C. J., \& Bate, M. R. 2006, MNRAS, 368, 1296

Bonnell, I., Martel, H., Bastien, P., Arcoragi, J.-P., Benz, W. 1991, ApJ, 377, 553

Bonnell, I. A., Smith, R. J., Clark, P. C., Bate, M. R. 2010, arXiv, 1009.1152

Boss, A. P., 1986, ApJS, 62, 519

Boss, A. P. \& Bodenheimer, P., 1979, ApJ, 234, 289

Boss, A. P., Fisher, R. T., Klein, R. I., McKee, C. F., 2000, ApJ, 528, 325

Brunt, C. M., Heyer, M. H., \& Mac Low, M.-M. 2009, MNRAS, 504, 883

Chapman, S., Pongracic, H., Disney, M., Nelson, A., Turner, J, Whitworth, A. 1992, Nature, 359,207

Clark, P. C. \& Bonnell, I. A., 2004, MNRAS, 347, 36

Clark, P. C., Bonnell, I. A., Zinnecker, H., Bate, M. R. 2005, MNRAS, 359, 809

Dobbs, C. L., Bonnell, I. A., \& Clark, P. C. 2005, MNRAS, 360, 2

Elmegreen, B.G., Klessen, R.S., \& Wilson, C.D. 2008, ApJ, 681, 365

Evans, N. J., Dunham, M. M., Jørgensen, J. K., Enoch, M. L., Merín, B., van Dishoeck, E. F., Alcalá, J. M., Myers, P. C., Stapelfeldt, K. R., Huard, T. L., Allen, L. E., Harvey, P. M., van Kempen, T., Blake, G. A., Koerner, D. W., Mundy, L. G., Padgett, D. L., Sargent, A. I. 2009, ApJS, 181, 321

Girichidis, P., Federrath, C., Banerjee, R., Klessen, R. S. 2010, arXiv, 1008.5255

Goldsmith, P. F., Heyer, M., Narayanan, G., Snell, R., Li, D., Brunt, C. 2008, ApJ, 680, 428

Hennebelle, P. \& Fromang, S. 2008, A\& A, 477, 9

Hennebelle, P. \& Teyssier, R. 2008, A\&A, 477, 25

Jappsen, A.-K., Klessen, R. S., Larson, R. B., Li, Y., Mac Low, M.-M. 2005, A\&A A, 435, 611

Klessen, R. S. 2001, ApJ, 550, 77

Klessen, R. S.\& Burkert, A. 2000, ApJS, 128, 287

Klessen, R. S. \& Burkert, A. 2001, ApJ, 549, 386

Klessen, R. S., Burkert, A., \& Bate, M. R. 1998, ApJ, 501, L205

Krumholz, M. R., Klein, R. I., \& McKee, C. F. 2007, ApJ, 656, 959

Krumholz, M. R., McKee, C. F., \& Klein, R. I. 2004, ApJ, 611, 399

Larson, R. B. 1969, MNRAS, 145, 271

Larson, R. B. 1972, MNRAS, 156, 437

Larson, R. B. 1981, MNRAS, 194, 809

Li, Z.-Y., Wang, P., Abel, T., Nakamura, F. 2010, ApJ, 720, L26

Offner, S. S. R., Klein, R. I., \& McKee, C. F. 2008, ApJ, 686, 1174

Offner, S. S. R., Klein, R. I., McKee, C. F., Krumholz, M. R. 2009, ApJ, 703, 131

Price, D. J. \& Bate, M. R. 2007, MNRAS, 377, 77

Price, D. J. \& Bate, M. R. 2008, MNRAS, 385, 1820

Price, D. J. \& Bate, M. R. 2009, MNRAS, 398, 33

Reipurth, B. \& Clarke, C. 2001, MNRAS, 122, 432

Urban, A., Martel, H., \& Evans, N. J. 2010, ApJ, 710, 1343 